\title{
LA FORMACIÓN DE MAESTROS DE EDUCACIÓN FÍSICA EN EL URUGUAY (1921-1930): JULIO J. RODRÍGUEZ1
}

\author{
Paola Dogliotti Moro \\ Universidad de la República, Montevideo.
}

\begin{abstract}
0380
FORMAÇÃO DE PROFESSORES DE EDUCAÇÃO FÍSICA

NO URUGUAI (1921-1930): JULIO J. RODRIGUEZ
\end{abstract}

Resumo

Este trabalho é parte de um conjunto mais amplo de indagações sobre as discursividades da formação, nas diversas formas que adquiriram a preparação de docentes de educação física no Uruguai, entre o final do século 19 e na primeira metade do século 20. São analisadas, especificamente, as formas de educação do corpo e a educação física no projeto de formação de professores de educação física ou de praças de esporte nos anos 1920, no âmbito da Comissão Nacional de Educação Física, por seu diretor técnico, Julio J. Rodriguez. Descreve-se as tensões e as ligações entre discursividades normalista, tecnicista e profissionalista.

Palavras-chave: discursividades, formação, corpo, educação física.

\section{THE PHYSICAL EDUCATION TEACHERS TRAINING IN URUGUAY (1921-1930): JULIO J. RODRIGUEZ}

\section{Abstract}

This work is part of a broader set of inquiries about training discursivities in the various forms which acquired the preparation of physical education teachers in Uruguay between the late nineteenth century and the first half of the twentieth century. Here we analyze specifically the ways of body education and especially the physical education in the proyect for the training of physical education teachers or plazas de deporte in the 20s, in the framework of the Physical Education National Commission, by its Technical Director, Julio J. Rodriguez. We describe the tensions and links between normalist, technicist and professionalist discursivities.

Key-words: discursivities, training, body, physical education.

\footnotetext{
${ }^{1}$ Este trabajo forma parte de la tesis de maestría titulada Cuerpo y curriculum: discursividades en torno a la formación de docentes de educación física en Uruguay (1874-1948) y se enmarca en la interacción de las líneas de investigación: Enseñanza Universitaria, Políticas educativas, currículum y enseñanza, ambas del Departamento. de Enseñanza y Aprendizaje, Instituto de Educación, y Políticas educativas, cuerpo y currículum, del Departamento de Educación Física y Prácticas Corporales del Isef, Udelar.

\begin{tabular}{|l|l|l|l|l|l|l}
\hline Hist. Educ. [Online] & Porto Alegre & v. 17 & n. 41 & Set./dez. 2013 & p. 139-158
\end{tabular}
}




\section{LA FORMACIÓN DE MAESTROS DE EDUCACIÓN FÍSICA EN EL URUGUAY (1921-1930): JULIO J. RODRÍGUEZ}

Resumen

Este trabajo forma parte de un conjunto de indagaciones más amplias sobre las discursividades de la formación en las diversas modalidades que adquirió la preparación de docentes de educación física en Uruguay entre fines del siglo 19 y la primera mitad del siglo 20. Aquí se analiza en forma específica los modos de la educación del cuerpo y en especial, la educación física en el proyecto de formación de maestros de educación física o de plazas de deporte en los años 20, en el marco de la Comisión Nacional de Educación Física, a cargo de su director técnico, Julio J. Rodríguez. Se describen las tensiones y acoplamientos entre las discursividades normalista, tecnicista y profesionalista.

Palabras-clave: discursividades, formación, cuerpo, educación física.

\section{FORMATION DES ENSEIGNANTS D'ÉDUCATION PHYSIQUE EN URUGUAY (1921-1930): JULIO J. RODRIGUEZ}

\section{Résume}

Ce travail fait partie d'un ensemble plus large de questions sur les discours de la formation dans les diverses formes qui ont acquis la préparation des professeurs d'éducation physique en Uruguay entre la fin du $19 \mathrm{e}$ siècle et la première moitié du $20 \mathrm{e}$ siècle. Nous analysons plus précisément les facons de l'éducation du corps et en particulier dans le projet d'éducation physique de formation professeurs d'éducation physique ou places de sports dans les années 20, dans le cadre de la Commission National D'education Physique, par son directeur technique, Julio J. Rodriguez. Nous décrivons les tensions et les liens entre discours normaliste, techniciste et professionaliste.

Mots-clé: discours, formation, corps, éducation physique. 


\section{Presentación}

L n este trabajo nos abocamos a realizar un análisis de los principales discursividades en torno a la formación de maestros de educación física presentes en Julio J. Rodríguez. Para esto tomamos, entre otras fuentes, el primer proyecto de formación de maestros de educación física del Uruguay del año 1921, que es de su autoría y que presentó a la CNEF desde su cargo de director técnico, cargo que ocupó desde el año 1920 a 1960. Es importante destacar su estrecha vinculación con Jess T. Hopkins ${ }^{2}$, en los agradecimientos realizados por Hopkins en su tesis de graduación, al único uruguayo que menciona es a Julio J. Rodríguez:

a mi querido amigo y discípulo Sr. Julio J. Rodríguez, quien ha tenido la bondad de criticar el castellano que he empleado y quien siempre me ha ayudado a discutir los temas en lo que se refiere a su manera de presentarlos para su uso en la América Latina. (Hopkins, 1918, p. 1)

Para Rodríguez fue su gran maestro y se declara su sucesor: "desde el retiro del prof. Hopkins ocupa la Dirección Técnica del Instituto el autor de esta reseña, graduado en la Universidad de Springfield. Fue su propósito continuar la obra del prof. Hopkins, que fuera su maestro" (Rodríguez, 1952, p. 6). Al referirse a las plazas de deportes Rodríguez expresa:

Fueron instaladas bajo la competente y autorizada dirección del Sr. Jess T. Hopkins. [...] Al iniciar su obra que tanto arraigo ha tomado entre nosotros, el Sr. Hopkins tuvo que vencer serios obstáculos que siempre encuentran los innovadores, hasta que con sus profundos conocimientos sobre la materia, la pureza de sus ideales, la corrección de sus procederes y la energía y bondad de su carácter, supo imponerse a la envidia, al prejuicio, a la indiferencia, a la ignorancia y a las malas intenciones. El personal enseñante de las primeras plazas de deportes fue preparado por Hopkins. (Rodríguez, 1923, p. 20)

Rodríguez continuó con la formación de maestros de educación física que su maestro había comenzado. Tuvo bajo su responsabilidad y dirección los cursos intensivos de preparación de maestros a partir del año 1923 hasta finales de la década del $30^{3}$. Fue seleccionado por Hopkins para realizar sus estudios universitarios en la Universidad de Springfield obteniendo el título de Bachiller en Educación Física (BPE) ${ }^{4}$. También fue miembro del Comité Olímpico Uruguayo (CNEF, 1924; Uruguay, 1929).

Entre sus principales escritos destacamos el primer Proyecto de Formación de maestros de educación física del Uruguay, publicado por la revista Uruguay-Sport en el

${ }^{2}$ Maestro de educación física por la universidad de Springfield College de la YMCA norteamericana. En 1912 se radicó en Uruguay para encargarse del Departamento de Educación Física de la ACJ Montevideo, fundado en junio de 1912. Entre 1913 y 1918 ocupó el cargo de director técnico de la recién creada CNEF (Comisión Nacional de Educación Física, fundada el 7 de julio de 1911, por decreto ley n. 3.798 , en la segunda presidencia de José Batlle y Ordóñez, dependía del Poder Ejecutivo, con el cometido de hacerse cargo de lo relativo a la promoción y desarrollo de la "cultura física" a nivel de todo el país), desde donde impulsó el establecimiento de plazas de deporte. Encargado del primer curso intensivo de formación de maestros de educación física desarrollado en el año 1920.

3 Para un análisis sobre la educación del cuerpo y las discursividades de la formación en estos cursos, consultar Dogliotti (2011).

${ }^{4}$ No continuó sus estudios para obtener el título de maestro de Educación Física sino que regresó a Uruguay para asumir la Dirección Técnica de la CNEF.

Hist. Educ [Online] Porto Alegre v. 17

\begin{tabular}{l|l} 
n. 41 & Set./dez. 2013
\end{tabular}

p. $139-158$ 
año 1921, y el primer Plan de Acción de la CNEF y Conclusiones que se derivan del mismo, escrito y editado por la CNEF en el año $1923^{5}$.

Julio J. Rodríguez asiste en representación de la CNEF al $3^{\text {er }}$ Congreso de Directores de Educación Física de las ACJ de Sudamérica, desarrollado en Piriápolis en febrero del año 1924 (Hopkins, 1927). Esto muestra las relaciones estrechas tejidas durante las dos primeras décadas de existencia de la CNEF entre este organismo y la YMCA.

\section{Educación física: degenerescencia, higienismo, carácter}

Desde un enfoque positivista y evolucionista eugenésico, al igual que Hopkins ${ }^{6}$, Julio J. Rodríguez concibe la educación física como una ciencia basada en las ciencias biológicas y psicológicas:

La educación física es una ciencia y sabemos que ésta no admite dogmas, ni principios absolutos, ni estrechez de escuelas. Ella está basada en las ciencias biológicas y psicológicas. Para aplicarla, primero se diagnostica y después se receta, y no como antes, que se aceptaban métodos y sistemas, como panaceas, que tanto se aplicaban a un niño en pleno crecimiento, como a un adulto que hacía vida sedentaria. (Rodríguez, 1923, p. 9)

La psicología es concebida desde un punto de vista biologicista y evolutivo, ya que es, según él, la ciencia que determina las características orgánicas y fisiológicas en cada etapa del desarrollo evolutivo desde el nacimiento hasta la vida adulta. El maestro de educación física debe estudiar al detalle todos estos cambios para adaptar "métodos y sistemas" a estas etapas. A esto dedica varias páginas de su plan de acción. En este sentido, podemos decir que el suelo epistémico ${ }^{7}$ desde donde se estructura a la educación física está constituido por las ciencias biológicas y los discursos sobre el cuerpo son los del atlas de anatomía y su funcionamiento, la fisiología.

Tal es así que concibe al músculo, al igual que Hopkins, como "órgano de la voluntad", "por medio del cual se realiza todo esfuerzo humano" (Rodríguez, 1923, p. 1). Desde este discurso, lo orgánico determina los modos de sentir, de pensar, y estructura al sujeto. Así, "el carácter puede ser definido como un plexo de hábitos motores" (Rodríguez, 1923, p. 2). Basándose en algunos autores pragmatistas norteamericanos, expresa:

Robertson cree que el hombre es lo que hace, vale decir, la suma de movimientos; la de Mandsley, que dice que el carácter es simplemente un conjunto de hábitos musculares; la de Blentschili que representa la historia como la agrupación de movimientos conscientes deseados; o la de Hall que dice que no podemos formarnos una concepción de fuerza o energía

\footnotetext{
${ }^{5}$ El plan fue aprobado en sesión del 17 de julio de 1923 y el resumen en agosto del mismo año (Rodríguez, 1923).

${ }^{6}$ Para un análisis sobre las concepciones sobre el cuerpo y la educación física en Hopkins referirse a Dogliotti (2012a).

7 Se entiende aquel "espacio de saber" y "configuraciones que han dado lugar a las diversas formas de conocimiento empírico". Se pregunta por "cuál espacio de orden se ha constituido el saber". Es el campo epistemológico en el que "los conocimientos, considerados fuera de cualquier criterio que se refiera a su valor racional o a sus formas objetivas, hunden su positividad y manifiestan una historia que no es la de su perfección creciente, sino la de sus condiciones de posibilidad" (Foucault, 1991, p. 7).
} 
sin la participación de nuestro esfuerzo muscular; todas estas expresiones, demuestran claramente cómo hemos modificado el antiguo concepto que de la vida tenía Cicerón al decir "vivere est cogitare" o "viver est velle" y sirven para orientarnos en el nuevo sentido de la importancia del desarrollo y régimen muscular. (Rodríguez, 1923, p. 2)

Desde un positivismo pragmatista el sujeto es determinado por el músculo, la ciencia es aquella que puede ser comprobable por medio de los sentidos, por lo observable, y a ésta la constituye, en última instancia, el músculo: "La psicología moderna considera a los músculos como órganos de expresión de los procesos eferentes [...] como órgano de la voluntad, sino también del pensamiento y del sentimiento" (Rodríguez, 1923, p. 2). Los músculos determinan los valores a ser adquiridos, "vehículos del hábito, la obediencia, la imitación, del carácter y aun de costumbres y maneras" (Ibid., p. 2).

Al igual que en el pensamiento de Hopkins, se establece una biologización de la moral o, incluso, podemos decir muscularización de la moral (Dogliotti, 2012a). Las ciencias biológicas prescriben normas de conducta; constituyen también el suelo epistémico de la psicología. También se musculariza o biologiza el cerebro: "el niño desarrolla su cerebro más por la acción de sus músculos que por el ejercicio de su intelecto" (Rodríguez, 1923, p. 3).

La educación física es, para Rodríguez, "una fuerza compensatoria" que viene a combatir la "degeneración de la raza humana"8 causada por los efectos de la civilización (Rodríguez, 1923, p. 6). A partir de la distinción mostrada por Vigarello (2005) entre degeneración y degenerescencia, más que de una degeneración en Rodríguez se hace referencia a aspectos que se acercan más a una teoría de la degenerescencia, ya que se refiere a la degeneración de la raza luego de las transformaciones ocurridas a lo largo del siglo 19 y los trastornos causados por el industrialismo, el desarrollo de las ciudades, entre otros. "Nosotros conocemos las condiciones actuales de nuestros habitantes, sabemos cuales son sus defectos, y sus necesidades y sabemos cuáles son los medios más eficaces para corregirlos y perfeccionarlos" (Rodríguez, 1923, p. 10).

Corrección y perfección son dos cualidades eugenésicas fundamentales para "contrarrestar las condiciones físicas degenerantes, y el medio anormal producido por la civilización y sus causas", para esto, el maestro de educación física "deberá sentirse animado por una gran esperanza ética y humanitaria, y un gran deseo para obtener una raza mejor" (Ibid., p. 41). La eugenesia es el principal ideal que otorga sentido a la tarea del maestro de educación física. La profesión se estructura desde esta matriz fundadora.

\footnotetext{
${ }^{8}$ Según Vigarello (2005), en la segunda mitad del siglo 18 el término degeneración predomina. La evolución cuantitativa de las poblaciones se presenta como un campo de objetivos, prácticas y saberes. Por primera vez la constitución física es considerada en un tiempo filogenético: "En el siglo XVIII, degeneración significa sobre todo degradación, debilitamiento, y en ningún sentido deslizamiento y modificación en la organización de los cuerpos. Nada hay que se parezca al transformismo o al evolucionismo en esa representación [...] Las desviaciones siguen siendo casi individuales y los rasgos primitivos siempre se pueden restituir una vez que se recupera el medio de origen. Nunca es la organización interna de los seres vivos la que desencadena los cambios, nunca esa estructura es portadora de historia" (Vigarello, 2005, p. 31). En la segunda mitad del siglo 19 la palabra degeneración es sustituida por degenerescencia. Es Morel, para quien los obstáculos para el desarrollo corporal inciden en el de la inteligencia, quien introduce el concepto de degenerescencia en relación con el de degeneración. "Es en la lectura de Morel, y pronto de Darwin, en la que se inspirarán hacia fines de siglo las teorías de la antievolución: la degenerescencia es el estado del ser que, contrariamente al de sus progenitores, se ve amenguado en su resistencia fisiológica" (Ibid., p. 110). Pero a diferencia del evolucionismo darwiniano, esta teoría es normativa. 
La educación física, según Rodríguez, debe perseguir cuatro grandes objetivos en cualquier tipo de institución, ya sea plaza de deportes, escuela, liceo, instituto correccional, cárcel, organización deportiva": "1. salud, 2. recreación física, 3. mejoramiento de la energía mental, 4. carácter" (Rodríguez, 1923, p. 10). El maestro de educación física es un colaborador del médico, ya que "el mejoramiento de la salud es una de las finalidades de la medicina y de la higiene. La medicina preventiva, día a día extiende su campo de acción, y es en este precisamente que la educación puede prestar grandes servicios" (Ibid., p. 10). Para esto el maestro debe fomentar el "crecimiento y desarrollo normal del organismo humano", "los exámenes físicos", "la gimnasia correctiva", que "desempeña un papel importante en la corrección de aquellos defectos posturales [...] causas de serias y desconocidas condiciones patológicas",

los principios y métodos higiénicos [...] para la divulgación sobre higiene personal. Sintetizando, se puede decir, que la educación física, puede contribuir a la obtención de aquellas condiciones que tienden, no sólo a la prolongación de la vida, sino también a su amplitud, vale decir, buena salud. (Rodríguez, 1923, p. 11)

La educación física se erige como la ciencia de la postura correcta, que elimina lo anormal, patológico, fuera de los estándares establecidos por las ciencias médicas. La higiene debe ser también profesada por el maestro de educación física. Así, "El discurso sobre la degenerescencia revela el lugar preeminente que adquiere la higiene. [...] La higiene viene a totalizar la red, cada vez más densa, de todo aquello que hace de la postura una prevención y un comportamiento" (Vigarello, 2005, p. 130). Uno de los blancos a los que apunta esta pedagogía higiénica es la pobreza:

En los barrios pobres de las ciudades las familias viven hacinadas en los conventillos; viviendas que deberían desaparecer por sus condiciones malsanas y antihigiénicas. [...] Es precisamente en estas gentes, donde las enfermedades contagiosas encuentran un campo fertilísimo. En lo referente a la moralidad, no podemos creer que de ella se tenga un alto concepto, viviendo en tales condiciones. (Rodríguez, 1923, p. 44)

La conducta antihigiénica es asociada a una cuestión de clase: los pobres, por ser pobres, no solo gozan del sello de la antihigiene sino de la antimoral. Moral e higiene se despliegan como dos caras de una misma moneda, normalizan a las poblaciones para combatir la degenerescencia. "Las campañas de higiene pública o personal" (Rodríguez, 1923, p. 46) son una buena herramienta para esto,

el dinero que los poderes públicos emplean en la educación física [...] producirá de inmediato grandes economías en las finanzas nacionales [...] previniendo enfermedades y vicios sociales. Cada plaza instalada, evita la construcción de un hospital y resta cientos de delincuentes a las cárceles y reformatorios. (Rodríguez, 1923, p. 47)

${ }^{9}$ El Plan de Acción del año 1923 proyecta la realización de educación física en las plazas de deportes, escuelas públicas, educación física universitaria, secundaria y preparatoria, en las facultades de enseñanza superior, en las dependencias del Patronato de Delincuentes y Menores, en las cárceles (Rodríguez, 1923). 
La educación física constituye una fuente de ahorro económico para el Estado. La recreación física, otro de los objetivos a lograr por parte de la educación física, es entendida como "desahogos y salidas apropiadas para la expresión de los instintos, que, de lo contrario, buscarán y hallarán salida en formas inconvenientes, no aceptables por la sociedad humana" (Rodríguez, 1923, p. 13), como "el café", "los prostíbulos", "la timba", "los juegos de azar", que han llevado "a tan lamentable estado de degeneración" (Ibid., p. 43). "La delincuencia infantil está basada en la represión del instinto del juego" (Ibid., p. 41), por eso la educación física es una herramienta esencial para su combate. El maestro de educación física canaliza los instintos sexuales en la etapa de la pubertad:

El desarrollo de los órganos sexuales, despierta en los pubescentes, nuevos instintos y deseos que deben ser bien dirigidos, por una persona, como por ejemplo, el maestro de educación física, que esté bien empapado de estas cuestiones, de lo contrario, ya encontrará ocasiones en rueda de amigotes o compañeros de mal vivir, para expresarlos en forma impropia y perjudicial para su salud. No olvidemos que la expresión de esos instintos y deseos en un ambiente malsano, puede convertir al niño en un pervertido sexual, en un criminal, o en fin, en un elemento poco recomendable para la sociedad. (Rodríguez, 1923, p. 116)

La nueva sensibilidad del siglo 20 (Barrán, 1994) trajo aparejada una visión sobre las conductas sexuales correctas y las prohibidas ${ }^{10}$. En este contexto, la educación física no solo era vista como un eficaz modo de represión sexual sino también sindical y político:

El Departamento de Educación Física será una gran escuela de disciplina y de moral. Las huelgas estudiantiles, tan generalizadas actualmente, no se sucederán tan frecuentemente, pues esos deseos y anhelos instintivos, que casi siempre forman parte de esos movimientos colectivos, podrán ser sublimados en el campo de juegos en una forma beneficiosa para ellos. (Rodríguez, 1923, p. 127)

En relación al cuarto objetivo de la educación física, Rodríguez señala que "el carácter" se desarrolla en el "campo de juego" ya que "es una excelente escuela moral". Allí se aprenden valores tales como: "cooperación" o "espíritu de solidaridad", "sacrificio personal para obtener el triunfo del equipo", "la cortesía que debe emplearse con sus compañeros y contrarios", "el coraje", "se aprende a tener mayor confianza en sí mismo" (Rodríguez, 1923, p. 14-15). Pareciera que todas estas cualidades están más referidas, sin explicitarlo, al sexo masculino. Esto queda evidenciado cuando Rodríguez, hace referencia a una conferencia de Marcel Prevost, de 1905, titulada El espíritu en la escuela de los deportes, en la que

demostró que las reglas del adiestramiento deportivo aplicadas al trabajo intelectual, pueden producir modestia, sinceridad, disciplina y otras buenas cualidades. El mismo autor en una de sus obras dice: "La asociación íntima de los ejercicios físicos y de los estudios profundos puede ser fecunda en explendores [sic] viriles". (Rodríguez, 1922, p. 3.701)

${ }^{10}$ Cuando se alude a las conductas sexuales se está refiriendo siempre, aunque no se explicite, al sexo masculino.

\begin{tabular}{|l|l|l|l|l|l|}
\hline Hist. Educ. [Online] & Porto Alegre & v. 17 & n. 41 & Set./dez. 2013 & p. 139-158
\end{tabular}


Para Rodríguez, los juegos y deportes son la actividad de preferencia a desarrollar en las plazas de deportes ${ }^{11}$, si bien la gimnasia ocupa su lugar de importancia, y "se sigue con algunas adaptaciones, las ideas del genial Ling, por entender que este fue el creador de la gimnástica" (Rodríguez, 1952, p. 9) ${ }^{12}$. Despliega todo un optimismo deportivo: para él, el deporte es una actividad humana "buena en sí misma", genera "libertad", "para que no se desviertúe su verdadera esencia, los juegos tienen que estar libres de cualquier coacción exterior o de cualquier necesidad interna" (Rodríguez, 1929, p. 39).

Este esencialismo se distancia de cualquier posibilidad de construcción histórica y social y también subjetiva. "Sabemos que los juegos son, por excelencia, los ejercicios que más placer y alegría nos proporcionan si son practicados de acuerdo con la propia naturaleza de los mismos" (Ibid., p. 40). Se presenta una naturalización de la práctica social del deporte a tal extremo que pareciera que la crítica cultural quedara suspendida. A partir de un supuesto mecanismo de selección natural darwiniana, se justifica e idealiza la pirámide selectiva social del deporte:

La idea de los Juegos Olímpicos del Barón de Coubertin, no tiende tan sólo, a la formación de un grupo limitado de campeones, para que cada cuatro años se luzcan demostrando sus habilidades y capacidades, sino también y como finalidad principal, a que las masas practiquen los deportes. Los campeones entonces, son el producto de una severa selección y el ideal que sirve de estímulo e incentivo para las muchedumbres. (Rodríguez, 1922, p. 3.702)

\section{Cuerpos masculinos y femeninos}

La generización de los cuerpos masculinos y femeninos ${ }^{13}$ se puede apreciar a lo largo de los textos de Rodríguez, quien recomienda actividades corporales adaptadas a cada uno de los sexos. Los hombres deben estar separados de las mujeres para realizar sus clases de educación física. Esto se muestra en la arquitectura de las instituciones y espacios diseñados para prácticas de educación física; por ejemplo, "una plaza de deportes bien instalada debe contar con dos grandes secciones, una para varones y otra para mujeres. Dentro de esta última debiera haber un espacio destinado a los niños de ambos sexos hasta seis años de edad" (Rodríguez, 1929, p. 43).

La arquitectura y el mobiliario tienen una acción performativa sobre los cuerpos, generizándolos de determinada manera y no de otra. La mujer es identificada con el cuidado de la edad infantil:

[en el] rincón infantil debiera haber aparatos de sube y baja, hamaquitas, sillas, donde las madres pudieran columpiar a sus hijitos; cajones de

\footnotetext{
${ }^{11}$ Propone también que en las plazas de deportes se desarrolle gimnasia (con fines higiénicos, correctivos, educativos y saludables), atletismo (la define como una práctica natural) y, para los días de lluvia, propone "trabajo mental" que comprende: música y canto, trabajo manual, estudio de la naturaleza, narración de cuentos, y danzas gimnásticas y regionales (Rodríguez, 1923, 1929).

${ }^{12} \mathrm{Al}$ realizar una breve reseña histórica de la educación física, dentro de los modernos destaca los sistemas gimnásticos alemanes y suecos. Luego menciona a los ingleses como iniciadores de los deportes y a los norteamericanos por "ocupar un sitial de honor entre las naciones del mundo que marchan a la cabeza de la educación física moderna" (Rodríguez, 1923, p. 8). A lo largo de sus textos se muestra la preferencia e influencia que ha ejercido Estados Unidos en su concepción de educación física.

${ }^{13}$ Para este apartado se siguen fundamentalmente los desarrollos de Scharagrodsky (2008, 2006a y 2006b) y Goellner (2008).

Hist. Educ. [Online]

Porto Alegre

v. 17

ก. 41

Set./dez. 2013

p. $139-158$
} 
arena. [...] En este lugar debiera haber bancos y espacios con sombra, para que en los días de mucho sol, las madres que llevan a sus pequeños pudieran sentarse cómodamente a tejer, leer o cualquier otra cosa. (Rodríguez, 1929, p. 43).

Los aparatos gimnásticos y de juegos también se diferencian según el sexo, "el caballo, las paralelas y el burro" (Ibid., p. 43), son exclusividad de los varones, ya que requieren demasiada fuerza muscular.

Los lugares de poder son desiguales: los hombres pueden llegar a ser directores y no así las mujeres, que tan solo pueden llegar al "rango" de maestra: "En la sección destinada a los varones, debiera haber un salón para el director de la plaza [...] En la sección mujeres, debería haber también un salón para la maestra" (lbid., p. 44).

En la Sección Universitaria (secundaria y preparatoria), Rodríguez diferencia por sexo la descripción de las etapas evolutivas de "la pubertad y la adolescencia". "Las niñas psicológicamente, tienen preferencia por determinadas actividades diferentes de las de los niños. [...] La niña [...] tiene por cada kilo de peso, poco más de las tres cuartas partes de la capacidad vital, que un niño de la misma edad" (Rodríguez, 1923, p. 118). Las características físicas son comparadas teniendo como punto ideal de referencia las medidas y habilidades del varón. La comparación se establece con criterios de jerarquización negativa hacia la mujer (por ejemplo, la capacidad vital es menor que la del varón):

La mujer es menos favorecida que el hombre en el desarrollo muscular, por eso ella no debe hacer ejercicios y esfuerzos tan grandes como los que aquel pudiera hacer. La fuerza de la mujer, medida con el dinamómetro representa apenas, dos tercios de la del hombre. Como hemos visto, en el momento de la pubertad, mientras el varón busca instintivamente las ocasiones de producir esfuerzos musculares intensos, la mujer por el contrario, se inclina por la calma y es más reservada. (Rodríguez, 1923, p. 121)

Las supuestas características biológicas prescriben las prácticas corporales, pero también conductas y características de personalidad. Se produce una biologización no solo de los tipos de ejercicios físicos, determinando algunos exclusivos para los hombres, sino también una biologización de la moral femenina y masculina. Pareciera que, lejos de ser una construcción cultural, son los instintos los que llevan a realizar esfuerzos musculares intensos. Se esencializa y naturalizan determinadas prácticas para cada uno de los sexos. Las supuestas características biológicas son imaginarizadas como "complejas", hasta al punto de asociarlas con la muerte: "el período de la pubertad de la mujer, tiende a ser más complicado que el del hombre, lo que se pone en evidencia al observar el alto grado de morbidez a tal edad" (Rodríguez, 1923, p. 119). La menstruación es vivida como un problema:

es conveniente que hasta que esta función no se haya regularizado, las niñas tengan mucho cuidado cuando ella ocurre, pues nadie mejor que las madres, saben bien, cuan peligrosas son las consecuencias de los fríos, de las mojaduras de pies o de los vestidos, etc. La higiene mental es tan necesaria en este período, como la higiene física. (Ibid., p. 120) 
El vestido es el atuendo de la mujer y los cuidados se extreman en esa etapa. La menstruación es identificada con la debilidad, donde "el sistema nervioso participa de la débil condición de todo el cuerpo y es por lo tanto susceptible de ser perturbado por cualquier desorden funcional" (Ibid., p. 120). Se presenta un único modo de generificación de los cuerpos de los hombres y las mujeres; no hay posibilidad de que coexistan diversas femineidades y masculinidades. Rodríguez expresa:

Es muy corriente, que muchos entiendan, que la mujer está capacitada para hacer los mismos ejercicios y juegos que el hombre y aconsejan por lo tanto el boxeo, la lucha, los ejercicios violentos, etc., que son de la preferencia del hombre. Esto es un grave error, pues la mujer no ha sido creada para pelear, no para hacer ejercicios de gran fuerza, sino más bien para realizar ese gran acto, tan sublime y tan necesario para la supervivencia de la raza humana, como lo es el de la procreación. Creemos que la mujer debe hacer ejercicios y juegos femeninos y no masculinos. [...] Los que se adaptan mejor a la mujer son lo que no exigen esfuerzos musculares intensos [...] ellos [...] pueden ser peligrosos. (Rodríguez, 1923, p. 121)

La construcción del cuerpo femenino es determinada a tal punto por la maternidad que no hay otra posibilidad de generificación de los cuerpos femeninos. Desde esta posibilidad de identificación se prescriben sus normas de conducta, y no así en el varón ${ }^{14}$. Las ciencias biológicas son las que justifican y otorgan supuesta neutralidad a las prescripciones sobre las conductas, específicamente sobre las prácticas corporales desde la única posibilidad que incardina (Scharagrodsky, 2006a) a la mujer a la maternidad. Pareciera ser su destino sagrado. Por esto,

recordemos que la mujer, después de la edad de la pubertad debe guardarse de ejecutar ejercicios y juegos que vayan acompañados de choques, encontrones y saltos violentos, pues pueden acarrear graves consecuencias, para el órgano uterino. La ciencia condena severamente tal práctica. (Rodríguez, 1923, p. 122)

\section{El maestro de educación física}

Para definir las principales características que debe poseer un maestro de educación física, Rodríguez recurre a citas de norteamericanos, la primera, "del ex-presidente de los Estados Unidos de Norte América, Sr. Teodoro Roosvelt”, quien, al referirse a la dirección de las plazas de deportes, sostiene:

es indispensable, de lo contrario los niños mayores o más fuertes, las ocuparían con exclusión de los menores o más débiles. Se tornarían tan ruidosas que los vecinos se verían molestados o bien servirían de sitio de reunión de elementos menos recomendables del vecindario. Por otra parte, los ejercicios y juegos serían menos sistemáticos y vigorosos si les faltara la dirección del personal competente. (Rodríguez, 1929, p. 45)

\footnotetext{
14 "Una de las principales razones de la incorporación femenina a las prácticas corporales fue la 'ideología de la maternidad' con la imagen de la mujer como 'guardiana de la raza'” (Scharagrodsky, 2006a, p. 165). Por el contrario, la propuesta de Rodríguez "en ningún momento menciona a la paternidad como el fin último de la Educación Física masculina. Vale decir, el hombre o el niño no fueron sinónimos de paternidad" (Scharagrodsky, 2006a, p. 172). \begin{tabular}{l|l} 
Hist. Educ. [Online] & Porto Alegre
\end{tabular} v. 17 ก. 41 Set./dez. 2013 p. $139-158$
} 
Se priorizan, por un lado, el ejercicio de la disciplina y, por otro, la experticia en el campo de los ejercicios y juegos físicos. Luego cita las palabras de la directora de una escuela de educación física norteamericana, la doctora Stoneroaf, quien expresa: "la personalidad del maestro, su voz y su manera de ser, inspiran el entusiasmo y facilitan la marcha de los juegos. La alegría y la felicidad son estados mentales que pueden reflejarse en los demás; el entusiasmo es contagioso" (Rodríguez, 1929, p. 45).

Se entiende que los aspectos actitudinales del maestro son centrales en su tarea. Rodríguez otorga relevancia a los atributos morales que debe poseer; por esto, la relación entre el maestro y el niño no debe ser similar a la que hay "entre el capataz y su cuadrilla de trabajadores", evitando que el maestro "ordenara imperativamente a los niños hacer tal o cual ejercicio o juego excluyendo la libertad y espontaneidad de éstos" (Rodríguez, 1929 , p. 45). En estos presupuestos podemos observar cierto escolanovismo: el centro es puesto en el niño y se prioriza la relación amistosa frente a la transmisión de conocimientos y la enseñanza. Esto lo expresa Rodríguez al citar al "Dr. M. Ángel, un ilustrado profesor de educación física", quien señala:

El maestro de la plaza de deportes no es necesariamente un maestro: es un jefe y mezclándose con los niños en sus juegos, los dirige más bien por la sugestión que por la enseñanza. Debe ser ingenioso y original, capaz de adaptarse a las varias circunstancias que puedan presentarse. Debe poseer tacto, ser considerado y estar siempre dispuesto para ayudar a los niños; ser un amigo de éstos y si consigue que lo miren con agrado y lo mezclen con gusto en sus juegos, puede estar satisfecho, pues ha llenado su misión de la manera más completa. (Rodríguez, 1929, p. 46)

Los fines altruistas caracterizan la misión del maestro, su personalidad y modos de ser son esenciales para esta tarea. Para esto son fundamentales los conocimientos de la psicología infantil. La discursividad psicológica (Bordoli, 2005) matriza a esta nueva profesión, como se lee en los escritos de Rodríguez:

Debe recordarse que los maestros de plazas de deportes, están en contacto directo con las manifestaciones y expresiones propias de la naturaleza infantil, y que para comprenderlas y guiarlas por un buen sendero, debieran conocer ante todo la psicología infantil, tanto o más que el maestro de escuela, y tener muy en cuenta que el niño todo lo imita y sigue siempre el ejemplo del "héroe". (1929, p. 46)

El maestro de educación física es el modelo a quien se debe imitar, y su tarea pedagógica es definida como la inculcación en el niño de los valores necesarios para la vida en sociedad. En las plazas de deportes, "el cigarro y el juego por dinero quedan proscritos y el muchacho se desarrolla bajo condiciones más benéficas para la salud física y moral". El maestro "modela el carácter de miles de niños en edad conveniente para conseguirlo y bajo condiciones poderosamente favorables" (Ibid., p. 47). Pero no solo es un "moldeador del carácter" sino también es definido como un "práctico" y "ejecutor". En palabras del Dr. M. Ángel, Rodríguez expresa:

El maestro debe conocer perfectamente a los niños, no teóricamente a través de las enseñanzas de la pedagogía y la psicología, sino por el recuerdo de su propia infancia, refrescada por el contacto directo con el niño. Debe poseer cualidades que lo hagan atrayente, ser discreto, atleta o 
gimnasta, porque nada conquista más el respeto de los muchachos que la fuerza muscular y la destreza física. Si no fuera atleta, debería en cambio poseer condiciones de jefe y un gran poder de apreciación con respecto a las necesidades del niño, de modo que pueda guiarlo en la dirección de su mayor interés. (Ibid., p. 46)

La fuerza muscular y la destreza física son puestas en un lugar de relevancia. En este sentido, únicamente el sexo masculino puede llegar a ser director de una plaza de deportes y la virilidad es expresada en la proeza de las acciones de fuerza. La matriz discursiva que atraviesa a la profesión es tecnicista: el maestro es un "organizador y director" de actividades (Ibid., p. 46).

A partir del modo de entender la profesión de Rodríguez, podemos decir que el polo técnico adquiere mayor relevancia que el polo psicológico. Las tradiciones normalista ${ }^{15}$ y la tecnicista son las predominantes, con mayor ascendencia, quizás, la primera sobre la segunda. En Hopkins esto se da de un modo similar aunque no tan pronunciado (Dogliotti, 2012a).

\section{Primer proyecto para la preparación de maestros de educación física en el Uruguay}

Preparación de Maestros de Educación Física en el Uruguay es el título que Julio J. Rodríguez le otorgó al primer proyecto sobre este rubro presentado a la CNEF en su cargo de director técnico. Fue aprobado por esta en la sesión 27 de diciembre de $1921^{16}$ y publicado en la revista Uruguay-Sport del mismo mes. Este proyecto implicaba una formación de un año de duración. Pasaron quince años antes de que se pudiera implementar: el curso de 1936 fue el único de un año de duración hasta la creación, en el año 1939, del Curso de Formación de Profesores de Educación Física de tres años de estudios.

Para elaborar y fundamentar su proyecto, Rodríguez se basó en el modelo norteamericano, fundamentalmente en el de la YMCA, que era en donde había realizado sus estudios de Bachiller en Educación Física. Sostiene:

Encontramos que, en épocas muy lejanas, en Alemania, Suecia, Dinamarca, Estados Unidos, etc. sus profesores de Gimnasia eran exacróbatas de circo, hombres de fuerza, levantadores de pesas, etc. Pero este modo de pensar ha cambiado gracias a los esfuerzos de ciertos hombres como: Ling en Suecia; Jhan en Alemania; Rousseau, Amorós, Lagrange y Tissié en Francia; Roberts, Sargent, Gulick, Fisher, Mac Curdy

\footnotetext{
${ }^{15}$ Rodríguez considera importante la preparación de los maestros de escuela para el dictado de clases de educación física. En su plan de 1923 propone un curso en la escuela normal de maestros; esta "debiera tener un Departamento de Educación Física bien organizado, a cargo de un buen director y los ayudantes que fueran necesarios. El Departamento de Educación Física e Higiene tendrá dos objetivos principales: 1- - La práctica de la educación física para bienestar de los propios alumnos, y 2-ㅡ. La preparación de estos alumnos por medio de cursos especiales, para poder dirigir inteligentemente las actividades del programa de educación física, que tendrán bajo su responsabilidad cuando terminen sus estudios normales y sean designados maestros" (Rodríguez, 1923, p. 109-110).

${ }^{16}$ Por resolución n. 7.880 del acta n. 20, que sostiene: "Apruébese en general, el Proyecto, presentado por la Dirección Técnica, sobre preparación de maestros de educación física. Desígnase una sub-Comisión formada por los Doctores Barbaroux y Ghigliani y Señores Monteverde, Hopkins y Rodríguez, con el cometido de resolver todo lo necesario para la aplicación del proyecto; y publíquese en la revista Uruguay-Sport, en el número correspondiente al mes de Diciembre actual" (Rodríguez, 1921, p. 3.127). 
en los Estados Unidos de Norte América, etc., y otros más que tuvieron la percepción de que la Educación Física no era sólo una serie de ejercicios o pruebas, sino una verdadera ciencia, basada en la psicología, higiene, anatomía, fisiología, sociología, etc. Muchos de ellos empezaron su carrera en un gimnasio bajo la dirección de uno de aquellos profesores, siguiendo después cursos de medicina. Por eso es que actualmente muchos de los dirigentes de la Educación Física, son médicos. En armonía con ese nuevo concepto es que desde hace algunas décadas, se han formado, especialmente en los Estados Unidos de Norte América, muchas escuelas y facultades de Educación Física, en las que se estudian las materias en igual forma que en cualquier otra facultad. Para ser admitido en ellas se necesita haber cursado bachillerato. Ya no es necesario que los graduados de estas facultades sigan cursos de medicina, pues, sus programas son vastísimos y están formados por materias que tienen íntima relación con la carrera de la Educación Física. Debido a esto, es que en los Estados Unidos de Norte América y en otros países, un director de Educación Física es considerado como cualquier otro titulado universitario. (Rodríguez, 1921, p. 3.142)

Las bases científicas de la educación física están puestas en la medicina. Según Rodríguez, los primeros que la practicaron fueron los médicos ya que los "acróbatas" de circo no merecían tal atributo científico sino que fueron las prácticas que la educación física necesitó relegar para constituirse como ciencia. "Parece que la Gimnasia Científica necesitó, para afirmarse como práctica corporal oficial de liberarse de sus orígenes impuros próximos al universo del arte, para crear una identidad, aún hoy tutelada por la medicina y la institución militar, la higiene y el espectáculo deportivo" (Soares, 2006, p. 19). Si bien en Estados Unidos el director de Educación Física es un titulado universitario, Rodríguez no estima necesario en Uruguay un instituto especial para su formación:

En primer término, sabemos que siendo nuestro país de población limitada, muy pronto podrá prepararse el número suficiente de maestros y maestras que sean necesarios para desarrollar un plan completo de Educación Física en toda la nación. Los gastos que demandaría la instalación de tal instituto así como los de su funcionamiento, serían de bastante consideración, lo que dificultaría el llevarlo a cabo y mantenerlo. Por otra parte, la experiencia de varios países sudamericanos, que establecieron institutos similares, no aconseja hacerlo aquí. (Rodríguez, 1921, p. 3.147)

Por estas razones, propone un "curso teórico-práctico de estudios, que a la vez fuera lo más completo y corto posible" (Ibid., p. 3.148). Como requisitos de ingreso se debe "haber terminado los estudios secundarios ${ }^{17}$, o los normales de primer grado, o, en su defecto, pasar por un examen de suficiencia" (Ibid., p. 3.148). También se recomienda "que los maestros que fueran a dirigir las actividades en estas instituciones, hubieran ellos mismos cursado los estudios en ellas" (Rodríguez, 1921, p. 3.148). El nivel de exigencia de ingreso es tan bajo - ya que mediante un examen de suficiencia ni siquiera se requería los cuatro años de secundaria completos - que pareciera que las condiciones físicas y morales del candidato están por encima de la preparación académica. Esto condice con la definición de Rodríguez para esta tarea como un apostolado que requiere de un sueldo discreto:

${ }^{17}$ Se refiere a los primeros cuatro años de secundaria, no incluyendo el bachillerato de dos años que era obligatorio para las carreras universitarias. Hist. Educ. [Online] $\quad$ Porto Alegre v. 17 \begin{tabular}{l|l} 
n. 41 & Set./dez. 2013
\end{tabular} p. $139-158$ 
Si la CNEF desea tener buenos maestros, conscientes, preparados, etc., es necesario que retribuya sus servicios en armonía con la calidad y cualidad exigidas. Si bien es cierto que el maestro o maestra deben mirar a la carrera que han escogido, como un medio para obtener el adelanto y progreso de nuestra raza y hacer de aquella un apostolado, no es justo ni es humano que lo hagan al precio de sacrificarse muchas veces, no sólo en perjuicio suyo, sino en el de su familia. [...] Debe ser recompensado con un sueldo discreto, que le permita vivir sin dificultades materiales". (Rodríguez, 1921, p. 3.147)

Los aspectos vocacionales y altruistas constituyen la matriz fundante de esta formación, que más que una profesión se presenta como una misión para el "adelanto y progreso de nuestra raza" (lbid., p. 3.147). Estos son aspectos que matrizaron tanto a la moral cristiana como a la laica. Desde ellos, Rodríguez destaca el sprit de corp que debería generarse entre los estudiantes del curso propuesto, propio del modelo cristiano de la YMCA de Estados Unidos:

Debiera ponerse especial atención en tratar de crear entre los estudiantes que siguieran este curso, un espíritu superior (sprit de corp) que los singularizara, es decir que fueran por su modo de proceder y actuar, fácilmente destacables de los demás. Es muy común oir hablar en los EE.UU. del espíritu de tal o cual universidad, y un estudiante o graduado de ellas tratará siempre de comportarse y actuar de acuerdo en él. No creo difícil la creación de este espíritu de cuerpo, ya que quienes seguirán el curso, serán personas seleccionadas. Además, he tenido conocimiento, que esto no será una experiencia nueva en nuestro país, pues según me ha informado, en la Escuela Naval, ya existe este "sprit de corp". (Ibid., p. 3.149)

En su dinámica gestacional, la formación de profesores estaba imbuida por estas características pastorales que no solo matrizaron diversas instituciones educativas con este sprit sino que en las militares también ocuparon su espacio.

En relación a los profesores que dictarían el curso, expresa:

Quizás uno de los problemas mayores que habrá que resolver es el de los profesores que tendrán a su cargo la enseñanza del programa de estudios del curso. Muchas de las materias que abarca, tales como: anatomía, fisiología, etc., podrán ser dictadas por algunos profesores de la Universidad. [...] Las materias que traten sobre la técnica de la Educación Física, deberán ser dictadas por profesionales titulados. Afortunadamente contamos en nuestro país con cierto número de graduados en Universidades extranjeras, que están relacionados con instituciones particulares $^{18}$, que podrán prestar sus servicios, si éstos le fueran requeridos". (Ibid., p. 3.150)

La mayoría de estos docentes eran graduados en las universidades de las YMCA estadounidenses. Con los textos ocurre algo similar:

${ }^{18}$ Se está refiriendo a la ACJ de Montevideo, que ha enviado a graduarse a Springfield a varios de sus líderes.

\begin{tabular}{|l|l|l|l|l|l|}
\hline Hist. Educ. [Online] & Porto Alegre & v. 17 & n. 41 & Set./dez. 2013 & p. 139-158
\end{tabular}


Otra de las dificultades [...] es la falta de bibliografía sobre Educación Física en castellano; muchos de los textos [...] tendrán que ser escritos expresamente o traducidos al castellano de otros idiomas, especialmente del inglés o francés. [... Habrá que] Preparar digestos o síntesis de algunos de ellos. (Ibid., p. 3.150)

El proyecto sugiere la entrega de un certificado, "en el que se hará constar" que "ha terminado el curso de un año de estudios ${ }^{19}$ organizados por la $\mathrm{CN}$ y que está capacitado para dirigir la Educación Física en cualquier establecimiento público de enseñanza, y en cualquier institución oficial o particular de la República" (Rodríguez, 1921, p. 3.150). La carga horaria diaria de clase es de cinco horas, "tres horas de enseñanza teórica y dos de enseñanza práctica" (Ibid., p. 3.151). A esto se agregan horas de "práctica de enseñanza": "los estudiantes deberán hacer cierto número de horas de práctica de enseñanza en las escuelas públicas, en la Sección de Enseñanza Secundaria y Preparatoria, en las Plazas de Deportes, etc." (Ibid., p. 3.150).

Según Rodríguez, el curso debe tener dos finalidades esenciales:

Primera - La de preparar técnicamente en teoría y práctica de Educación Física a maestros y maestras para plazas de deportes, liceos de enseñanza secundaria, escuelas públicas, gimnasios, etc. Segunda - La de formar maestros y maestras de Educación Física que sean verdaderos "leaders" de la juventud, no solamente en la parte física sino en la parte social y moral. (Rodríguez, 1921, p. 3.151).

La tecnología pastoral ${ }^{20}$ vuelve a aparecer. Los maestros de educación física son, impulsados por el sprit de la YMCA, verdaderos leaders, pastores del rebaño juvenil que deben conducir social y moralmente.

El programa del Curso de Educación Física se divide en una parte práctica y una teórica y está distribuido en tres trimestres. La carga horaria del curso es de 80 horas semanales, con 54 horas dedicadas a la parte teórica y las restantes 36 horas a las materias prácticas (Ibid., p. 3.159). Este modo de división de las materias en prácticas y teóricas da cuenta del énfasis puesto en la ejecución: el maestro de educación física debía ser, primero que nada, un buen ejecutor y demostrar con el ejemplo los ejercicios a enseñar. No se concebía la posibilidad de que las materias prácticas, específicas de las diversas prácticas corporales, pudieran desarrollar aspectos teóricos y trascender los aspectos de ejecución.

A continuación se detallan cada una de las materias comprendidas en el parte teórica y su carga horaria, agrupadas según un criterio temático disciplinar - en el grupo 1 las ciencias biológicas y médicas, en el grupo 2 las ciencias de la administración, y en el grupo 3 una materia que pertenece al área específica de la educación física:

1) Anatomía y Kinesiología (144 horas), Fisiología (72 horas), Biología (60 horas), Fisiología del Ejercicio (48 horas), Antropología y Exámenes Físicos (36 horas), Higiene

${ }^{19}$ Un año escolar de estudios, un período continuo de nueve meses, desde marzo a noviembre inclusive (Rodríguez, 1921, p. 3.151).

20 "El cristianismo no dejó su huella indeleble en la escuela [...] En lugar de eso, fue la fuente de una tecnología pedagógica específica, de un conjunto especial de "disciplinas espirituales" (de una práctica particular de relacionarse y gobernarse a sí mismo) personificada en la relación pastoral entre el maestro y el alumno" (Hunter, 1998, p. 23). 
Individual (36 horas), Higiene pública y escolar (36 horas), Masajes y Primeros Auxilios (24 horas). Total: 456 horas.

2) Juegos y Plazas de Deportes (102 horas), Organización y Administración del Departamento de Educación Física (36 horas). Total: 138 horas.

3) Historia de la Educación Física (48 horas).

Se destaca un currículum centrado en las ciencias médicas. Las materias de esa área ocupan $71 \%$ del total de las asignaturas teóricas. A su vez, en varias unidades de las materias reunidas en el grupo 2, la salud y la higiene forman parte de ellas al igual que en Historia de la Educación Física, donde

el estudio de esta materia será el de dar al estudiante un bosquejo del origen y desarrollo de la Educación Física como ciencia, para hacer ver la importancia de la salud nacional por medio de un estudio de las causas que contribuyeron al éxito o fracaso en los pueblos antiguos. (Rodríguez, 1921, p. 3.155)

Las materias reunidas en el grupo 2 están nucleadas por poseer ciertos componentes de las ciencias de la administración, aunque en mucho menor grado que en los programas de las universidades de las YMCA norteamericanas. En la materia Organización y Administración de la Educación Física, "se estudiarán los planes de organización y dirección del Departamento de Educación Física e Higiene" de diversas instituciones, "el modo de formar instructores y ayudantes voluntarios, la organización de campeonatos, concursos, festivales, demostraciones, etc." (Rodríguez, 1921, p. 3.156). En la materia El juego y las plazas de deportes dos de sus seis unidades son dedicadas a temas administrativos, referidas una a dirección y la otra a organización de plazas de deportes. Entre sus temas se detallan: "cuidado de aparatos", "planeamiento", "estudio de las facilidades", "construcción", "equipo", "oficina" (Ibid., p. 3.157).

Es claro el sentido utilitario y pragmático de estas materias. Las restantes bolillas de esta materia están dedicadas a "naturaleza, función y filosofía del niño y del juego", con un enfoque evolucionista. Entre los temas destacamos: "relación entre la teoría de la evolución y la vida animal", "relación entre la teoría de la evolución y el hombre", "teoría que considera a la niñez como la recapitulación de la historia de la raza humana", "leyes generales de la herencia", "períodos de la niñez" (lbid., p. 3.156).

Las ciencias biológicas también permean a esta materia y constituyen su suelo epistémico. Las escasas referencias a la psicología tienen esta procedencia, lo orgánico y evolutivo matriza los cuerpos y sus miradas. Pareciera no haber posibilidad para otro saber que no sea el de las ciencias naturales. Desde aquí se edifica la mirada sobre lo social y se atribuye a los objetos de las ciencias humanas y sociales esencialidades 0 naturalizaciones, olvidando que los cuerpos son también producidos social y culturalmente. Desde las ciencias biológicas se prescriben juegos con "diferencias entre la edad y el sexo", "diferencias masculinas y femeninas" y se establecen estándares y criterios de normalización (por ejemplo, Rodríguez habla de "el juego de los niños anormales") (Rodríguez, 1921, p. 3.157).

Las poblaciones son clasificadas, nombradas y separadas en diversas categorías según: edad, sexo, inteligencia (normal-anormal). Las ciencias biológicas son las "verdaderas" ciencias que dictan normas de crecimiento y de conducta, "el juego y el 
crecimiento físico", "el juego y el crecimiento moral", "el instinto y la tradición del juego" (lbid., p. 3.157).

La pedagogía y la psicología no tienen presencia en el Programa, ni como materias ni como unidades en alguna de ellas. Dentro de los temas reunidos en las unidades relativas a "filosofía" y "naturaleza" del juego hay solo uno dedicado al "juego y la educación", y otros dedicados al "instinto y la tradición del juego", "la niñez en general y en particular. Períodos de la niñez" y "factores que controlan la expresión del impulso del juego" (lbid., 3.156). Los esbozos de cierta psicología están sostenidos por las ciencias biológicas.

La "parte práctica" no está presentada por materias como "la teórica" sino que se divide en tres grupos de actividades corporales, ordenadas según el tipo de lugar utilizado: en el gimnasio, al aire libre y en el agua (lbid., p. 3.158). La gran cantidad de actividades corporales que eran propuestas en la "parte práctica" da cuenta del énfasis dado a la ejecución y no tanto a la práctica de la enseñanza. Lo que identificaba al maestro de educación física en este programa es su sentido utilitario y práctico ligado a la ejecución de infinidad de ejercicios.

\section{Consideraciones finales}

Las principales discursividades en relación a la formación del maestro de educación física presentes en Julio Rodríguez, son la normalista y la tecnicista.

La primera, de un modo similar a como se presenta en Varela, el Congreso Pedagógico, Lamas, Ghigliani, y Hopkins ${ }^{21}$, da cuenta de una propuesta curricular de formación imbuida de aspectos morales, normas de conducta, donde lo vocacional y el espíritu altruista constituyen el eje de la formación del maestro de plaza de deportes, tanto en los mecanismos de selección de los candidatos, en el sprit de corp a generar y en los contenidos de las asignaturas como en la centralidad de los aspectos de disciplina a inculcar en los niños, aunque se realice de un modo amistoso. Una diferencia respecto de la discursividad normalista presente en Varela ${ }^{22}$ y en el Congreso Pedagógico ${ }^{23}$ es que la tradición psicológica aparece de forma bastante más moderada. Esto se aprecia en la ausencia en la malla curricular del proyecto de Julio Rodríguez de asignaturas y unidades relativas tanto a las diversas psicologías como a la pedagogía.

La discursividad tecnicista se presenta en los escritos de Julio Rodríguez más moderada que en los programas de las universidades norteamericanas de las YMCA y en Hopkins, pero más acentuada que en los discursos varelianos y los presentes en el Congreso Pedagógico. En este sentido, en relación a las ciencias de la administración, en la malla curricular del proyecto de Rodríguez, a diferencia del Programa de Maestros de

${ }^{21}$ Para un análisis en estos autores de las discursividades en torno a la formación de docentes, y en especial de educación física, consultar Dogliotti (2012b). Todos ellos ocuparon un rol protagónico en la formación de docentes, los dos primeros (Varela y el Congreso Pedagógico) en relación a la instauración del sistema educativo moderno uruguayo, los tres últimos en la formación de docentes de educación física.

${ }^{22}$ En relación a este tema consultar Rodríguez Giménez (2012), Bordoli; Dogliotti (2012).

${ }^{23}$ Para análisis de las discursividades de formación presentes en este congreso, se puede referir a Dogliotti (2012c).
Hist. Educ. [Online]
Porto Alegre
v. 17
n. 41
Set./dez. 2013
p. $139-158$ 
$1910^{24}$, hay materias específicas. En cierta medida, podríamos decir que la influencia de la perspectiva pragmática de la administración tayloriana tuvo mayor ascendencia en la formación de docentes de educación física. Aunque esta tendencia es más atenuada que la presente en la malla curricular de la universidad de la YMCA.

A lo largo de los escritos de Julio J. Rodríguez, la matriz profesionalista es más que débil, la educación es vista más como una misión y una vocación que como profesión. Esto, junto a la escasez de recursos económicos, puede haber sido uno de los motivos por los cuales se haya demorado tantos años para la creación de una carrera con otorgamiento de título ${ }^{25}$. A diferencia de las discursividades en torno a la formación de las universidades norteamericanas, en Uruguay primó lo pedagógico-normalista sobre lo profesional-económico-administrativo, que en Norteamérica fue uno de los motores del crecimiento exponencial de la profesión de director físico, a diferencia de lo ocurrido en un país menos desarrollado económicamente, como Uruguay.

\section{Referencias}

BARRÁN, José Pedro. Historia de la sensibilidad en el Uruguay. Montevideo: Banda Oriental, 1994.

BORDOLI, Eloísa La Didáctica y lo didáctico. Del sujeto epistemológico al sujeto del deseo. In: BEHARES, Luis; COLOMBO, Susana (comps.). Enseñanza del saber y saber de la enseñanza. Montevideo: FHCE, 2005, p. 17-25.

BORDOLI, Eloísa; DOGLIOTTI, Paola. Una mirada sobre la formación de maestros en Uruguay. Revisitando sus discursos: aproximaciones al normalismo. In: CORIA, Adela; REGUERA, Alejandra (comp.). ENCUENTRO INTERNACIONAL DE INVESTIGADORES DE POLÍTICAS EDUCATIVAS, 5, 2012, Córdoba. Actas ... Córdoba: AUGM-Nepi-PPE, 2012, p. 62-69.

CNEF. Informes, horarios y otros detalles del tercer curso intensivo teórico-práctico para la preparación de maestros de educación física a realizarse en Piriápolis del 17 al 29 de Marzo de 1924. Montevideo: CNEF, 1924.

DOGLIOTTI, Paola. Cuerpo y curriculum: el período de indefinición de la formación de profesores de Educación Física en Uruguay (1920-1936). In: ROZENGARDT, Rodolfo; ACOSTA, Fernando (comps.). Historia de la educación física y sus instituciones: continuidades y rupturas. Buenos Aires: Miño y Dávila, 2011, p. 231-257.

DOGLIOTTI, Paola. La influencia de la YMCA en el proceso gestacional de la CNEF en el Uruguay: Jess T. Hopkins. ENCUENTRO NACIONAL Y INTERNACIONAL DE INVESTIGADORES EN EDUCACIÓN FÍSICA, 16, 2012, Montevideo. Actas ... Montevideo: Isef, 2012a, en prensa. Disponible en: <www.isef.edu.uy $>$. Acceso en: 21 nov., 2012.

DOGLIOTTI, Paola. Cuerpo y curriculum: discursividades en torno a la formación de docentes de educación física en Uruguay (1874-1948). Montevideo: FHCE, 2012b. 328f. Tesis (maestría en Enseñanza Universitaria). Área Social. Comisión Sectorial de Enseñanza. Universidad de la Republica, Uruguay.

\footnotetext{
${ }^{24}$ Primer programa donde se incluye educación física como bolilla teórica en la asignatura de pedagogía, y gimnástica y juegos libres como asignatura práctica para todos los años de la formación magisterial. Para un análisis de este programa consultar Dogliotti (2012b).

${ }^{25}$ Se crea recién en el año 1939, en el marco de la CNEF, fuera de la órbita universitaria y no se requerían los años de bachillerato para su ingreso.

Hist. Educ. [Online] $\quad$ Porto Alegre

v. 17

ก. 41

Set./dez. 2013

p. $139-158$
} 
DOGLIOTTI, Paola. La figura del maestro en los albores de la tradición psicológica: el Congreso Pedagógico Internacional Americano, de 1882. Políticas Educativas, Porto Alegre, v. 5, n. 1, p. 59-77, 2012c. Disponible em: <http://seer.ufrgs.br/Poled/article/view/ 35733/23235>. Acceso en: 21 nov., 2012.

FOUCAULT, Michel. Las palabras y las cosas. México: Siglo 21, 1991.

GOELLNER, Silvia. El deporte y la cultura fitness como espacios de generificación de los cuerpos. In: SCHARAGRODSKY, Pablo (comp.). Gobernar es ejercitar: fragmentos históricos de la educación física en iberoamérica. Buenos Aires: Prometeo, 2008, p. 137152.

HOPKINS, Jess T. A monograph on physical education written in Spanish. Graduation Thesis by Jess T. Hopkins. International Young Men's Christian Association College. Springfield, Massachusetts, 1918.

HOPKINS, Jes T. Quince años de educación física en las asociaciones de América del Sur (1911-1926). Montevideo-Buenos Aires: Mundo Nuevo, 1927.

HUNTER, lan. Repensar la escuela: subjetividad, burocracia y crítica. Barcelona: Pomares-Corredor, 1998.

RODRÍGUEZ, Julio J. Proyecto de preparación de maestros de educación física en el Uruguay. Uruguay-Sport. Archivos de la Comisión Nacional de Educación Física, n. 48, Montevideo: CNEF, 1921, p. 3141-3159.

RODRÍGUEZ, Julio J. ¿Qué es el olimpismo? In: Uruguay-Sport. Archivos de la Comisión Nacional de Educación Física, n. 54, Montevideo, Uruguay, 1922, p. 3701-3702.

RODRÍGUEZ, Julio J. Plan de acción de la comisión nacional de educación física y conclusiones que se derivan del mismo. Montevideo: CNEF, 1923.

RODRÍGUEZ, Julio J. Plazas de deportes. In: Reglamentos oficiales de juegos atléticos y estudios sobre educación física moderna. Buenos Aires-Montevideo: Mundo Nuevo, 1929, p. 33-47.

RODRÍGUEZ, Julio J. Educación física en el Uruguay. Montevideo: CNEF, 1930.

RODRÍGUEZ, Julio J. Una reseña histórica de la educación física y la recreación en el Uruguay. Montevideo: CNEF, 1952.

RODRÍGUEZ GIMÉNEZ, Raumar. Saber del cuerpo: una exploración entre normalismo y universidad en ocasión de la educación física (Uruguay, 1876-1939). Montevideo: FHCE, 2012. 262f. Tesis (maestría en Enseñanza Universitaria). Comisión Sectorial de Enseñanza. Universidad de la Republica.

SCHARAGRODSKY, Pablo El padre de la educación física argentina: fabricando una política corporal generizada (1901-1938). In: AINSENTEIN, Ángela (comp.). Cuerpo y cultura: prácticas corporales y diversidad. Buenos Aires: Libros del Rojas, 2006a, p. 159197.

SCHARAGRODSKY, Pablo. Construyendo masculinidades y feminidades católicas y moralmente correctas: el caso de la primera Dirección General del Educación Física Argentina (1936-1940). In: AINSENTEIN, Ángela (comp.). Cuerpo y cultura: prácticas corporales y diversidad. Buenos Aires: Libros del Rojas, 2006b, p. 199-234.

SCHARAGRODSKY, Pablo. Entre la maternidad y la histeria. Medicina, prácticas corporales y feminidad en el Buenos Aires del fin del siglo 20. In: SCHARAGRODSKY, Pablo (comp.). Gobernar es ejercitar: fragmentos históricos de la educación física en iberoamérica. Buenos Aires: Prometeo, 2008, p. 105-135. 
SOARES, Carmen. Prácticas corporales: historias de lo diverso y lo homogéneo. In: AINSENTEIN, Ángela (comp.). Cuerpo y cultura: prácticas corporales y diversidad. Buenos Aires: Libros del Rojas, 2006, p. 9-36.

URUGUAY. Reglamentos oficiales de juegos atléticos y estudios sobre educación física moderna. Buenos Aires-Montevideo: Mundo Nuevo, 1929.

VIGARELLO, Georges. Corregir el cuerpo: historia de un poder pedagógico. Buenos Aires: Nueva Visión, 2005.

PAOLA DOGLIOTTI es profesora de la Universidad de la República, Uruguay. Magíster en enseñanza universitaria.

Dirección: Maturana 953 - Montevideo, CP 11700 - Uruguay.

E-mail: paoladogliottimoro@gmail.com.

Recebido em 26 de novembro de 2012.

Aceito em 17 de abril de 2013. 